\title{
A combined speckle tracking and phase-shift estimation approach for 2D blood flow imaging in the carotid bifurcation
}

\author{
Abigail Swillens \\ Patrick Segers \\ Institute Biomedical Technology-bioMMeda \\ Ghent University, Ghent, Belgium \\ Email: abigail.swillens@ugent.be
}

\author{
Lasse Lovstakken \\ Department of Circulation and Medical Imaging \\ NTNU, Trondheim, Norway \\ St. Olavs University Hospital, Trondheim, Norway
}

\begin{abstract}
A 2D blood velocity estimator is presented combining speckle tracking (ST) and phase-shift estimation (PE) to measure lateral $\left(v_{x}\right)$ and axial $\left(v_{z}\right)$ velocities respectively. Estimator properties were assessed in a computer model of a carotid bifurcation using ultrasound simulations based on computational fluid dynamics, allowing validation towards a ground truth. Simulation results were supported with in-vivo data of a healthy carotid. ST and PE estimates were combined as: 1) $v_{x}$ from 2D-ST and $v_{z}$ from PE, 2) $v_{x}$ from 2D-ST and $v_{z}$ from PE with aliasing correction based on ST, 3) $v_{z}$ from PE and only lateral ST for $v_{x}$. Regression analysis showed a 35-77\% decrease in standard deviation for $v_{z}$ for PE compared to ST. Aliasing correction based on ST improved results but also introduced spurious artifacts. A marginal decrease in performance was observed when only tracking laterally.
\end{abstract}

\section{INTRODUCTION}

Ultrasonic blood flow imaging is commonly applied in clinical practice, although still mainly limited to 1D Dopplerrelated techniques. Since complex flow fields are present throughout the arterial system, multidimensional flow imaging is desirable. This would allow better assessment of the flow field and its associated hemodynamic parameters, and hence potentially improve cardiovascular risk assessment.

Extensive research has been done on 2D flow imaging, mainly focusing on speckle tracking (ST) and vector Doppler (VD). The former relies on tracking the movement of speckle patterns, created by the interference of the ultrasonic waves backscattered by the red blood cells. The latter is the natural extension of 1D Doppler techniques, insonifying the blood vessel in two different directions, allowing construction of the velocity vector through triangulation. The performance of these estimators has been analyzed for analytically described flow patterns as well as under in-vitro and in-vivo flow conditions. However, to thoroughly investigate these techniques, accurate knowledge on the actually imaged flow field is indispensable. Therefore, we developed an ultrasound (US) simulation environment based on computational fluid dynamics (CFD), to allow simulation of ultrasonic images originating from complex flow fields, which can be validated towards a ground truth [1]. We previously used this multi- physics environment to compare estimator properties of ST and VD [2]. We demonstrated ST to be the better lateral (perpendicular to US beam) velocity estimator and VD the better axial (parallel to US beam) estimator.

In this work, we present a natural extension of these results by combining ST and phase-shift estimation (as used in VD) in one 2D flow estimator. A single scan procedure is used, and the RF-signals are processed with an ST-algorithm for the lateral flow component and with a PE-method for axial flow. In a first step, we analyze the combined estimator in a carotid bifurcation using CFD-based ultrasound simulations, allowing direct comparison of the estimated flow with the reference CFD flow field. Finally, in-vivo data measured in the carotid bifurcation of a healthy volunteer will be analyzed using our different estimator setups to support simulation results.

\section{Methods}

\section{A. Data acquisition}

Frame rate was maximized with a beam interleaved acquisition, ensuring that the waiting time between beams is minimized when the Doppler PRF is chosen lower than the maximum possible PRF ( $\propto$ image depth). This divides the image into lateral subregions (interleavegroups) where neighboring beams are acquired at $P R F_{\max }$, while a given beam direction is acquired at Doppler PRF. The number of beams in an interleavegroup (IGS) is: $I G S=\left\lfloor\frac{P R F_{\max }}{P R F}\right\rfloor \cdot P R B$ with PRB the number of beams received in parallel.

\section{B. $2 D$ velocity estimation}

1) PE: The autocorrelation method for phase-shift estimation was applied, as given by: $v_{z}=\frac{c P R F}{4 \pi f_{0}} \operatorname{atan}\left(\frac{\operatorname{Im}(\hat{R}(1))}{\operatorname{Re}(\hat{R}(1))}\right)$, with $v_{z}$ the axial velocity, $\mathrm{c}$ the ultrasound wave speed, $f_{0}$ the center frequency of the ultrasound pulse and $\hat{R}(1)$ the estimated autocorrelation function at lag 1. A 2nd order polynomial regression filter was applied prior to PE.

2) ST: A best match of a kernel region in a first image acquisition was searched for inside a search region of a later acquisition. The match between the kernel inside the search 
region was determined using the sum-of-absolute differences (SAD) algorithm:

$$
\epsilon(\alpha, \beta, n)=\sum_{i=1}^{l} \sum_{j=1}^{k}\left|X_{0}(i, j)-X_{n}(i+\alpha, j+\beta)\right|
$$

with $\epsilon$ the SAD coefficient, $l$ and $k$ the lateral and axial dimension of the kernel, $(\alpha, \beta)$ a kernel displacement in the lateral and axial direction, and $n$ the lag between acquisitions used for tracking. Using the kernel displacement corresponding to the minimal SAD-value $\left(\alpha_{m}, \beta_{m}\right)$, the velocity magnitude and angle can be estimated:

$$
V_{n}=\frac{\sqrt{\left(\alpha_{m} \Delta x\right)^{2}+\left(\beta_{m} \Delta z\right)^{2}}}{n T}, \Theta_{n}=\operatorname{atan} \frac{\alpha_{m} \Delta x}{\beta_{m} \Delta z},
$$

with $\Delta x$ and $\Delta z$ the lateral and axial sampling distances and $T$ the time between subsequent acquisitions. The procedure was performed for individual lags $(\mathrm{n}=1)$ in a packet of speckle images. The median of these estimates was calculated to minimize the effect of spurious tracking errors. We interpolated the image in both the axial $(1 \mathrm{x})$ and lateral $(3 \mathrm{x})$ directions and further parabolically interpolated the SAD matrix to achieve subpixel accuracy. Further, a 4th order FIR filter was used prior to ST.

3) Combining ST and PE: The same scanning procedure was applied for ST and PE, and two different imaging setups were investigated: a PRF of $2 \mathrm{kHz}$ and $4 \mathrm{kHz}$. Further, a $\mathrm{PRF}_{\max }$ of $16 \mathrm{kHz}$ and a transducer centre frequency of 5 $\mathrm{MHz}$ was applied, two receive lines were acquired in parallel, and a packet size of 10 was acquired for clutter filtering and averaging the estimated flow velocities. Using this setup, the autocorrelation method resulted in a maximal measurable velocity of $15 \mathrm{~cm} / \mathrm{s}$ and $31 \mathrm{~cm} / \mathrm{s}(\mathrm{PRF}=2$ and $4 \mathrm{kHz})$. For $\mathrm{ST}$, an interleavegroup size of 16 and 8 beams was obtained for respectively 2 and $4 \mathrm{kHz}$. The lateral beam density was close to the Rayleigh criterion for both setups, and resulted in 9 and 18 interleave groups $(\mathrm{PRF}=2$ and $4 \mathrm{kHz})$, and an overall frame rate of 22 fps.

For ST, a kernel size of $0.88 \times 0.52 \mathrm{~mm}$ (lateral $\mathrm{x}$ axial) was chosen, resulting in a maximal trackable velocity of $85 \mathrm{~cm} / \mathrm{s}$ and $65 \mathrm{~cm} / \mathrm{s}$ in respectively the lateral and axial direction. This choice corresponded with a search region of $1.36 \mathrm{x}$ $1.53 \mathrm{~mm}$ for $2 \mathrm{kHz}$ and $0.94 \times 1.14 \mathrm{~mm}$ for $4 \mathrm{kHz}$. Two kernels fitted in the $2 \mathrm{kHz}$ interleave region and one kernel in the $4 \mathrm{kHz}$ interleave region. After envelope interpolation, the velocity resolution for ST, prior to parabolic interpolation, was $7 \mathrm{~cm} / \mathrm{s}$ and $14 \mathrm{~cm} / \mathrm{s}$ laterally $(P R F=2$ and $4 \mathrm{kHz}$ ). In the axial direction, the resolution velocity was $3 \mathrm{~cm} / \mathrm{s}(2 \mathrm{kHz})$ and $6 \mathrm{~cm} / \mathrm{s}(4 \mathrm{kHz})$.

Three different possibilities of combining the lateral velocity $v_{x}$ from ST $\left(v_{x, S T}\right)$ and axial velocity $v_{z}$ from $\mathrm{PE}\left(v_{z, P E}\right)$ were investigated: (1) $v_{x, S T}$ from 2D-ST and $v_{z, P E}$ are combined by replacing the axial estimate of ST by the phaseshift estimate, further labeled as ST-PE,

(2) $v_{x, S T}$ from 2D-ST and $v_{z, P E}$ are combined but $v_{z, P E}$ is corrected for aliasing artifacts based on $v_{z, S T}$ :

$$
\angle \hat{R}(1)_{\text {corrected }}=\angle \hat{R}(1)+\operatorname{sign}\left(v_{z, S T}\right) \cdot\left\lfloor\left|v_{z, S T}\right| / v_{N y q u i s t}\right\rfloor \cdot 2 \pi
$$

with $\angle \hat{R}(1)$ the angle of the autocorrelation function at lag 1 and $v_{N y q u i s t}$ the Nyquist velocity limit. This method is further labeled as ST-PE: unwrapped,

(3) a simplified speckle tracking where the axial kernel displacement corresponds to $v_{z, P E}$ while the search region is confined to lateral tracking (1D-ST) only. This method is further labeled ST-simplified.

The variance of the velocity estimates was reduced through spatial averaging: (1) PE-estimates were averaged in a region of 3 beams and 2 pulselengths, (2) estimates from ST and the combined ST-PE methods were median filtered in a $5 \times 3$ kernel region.

4) Performance analysis: A linear regression analysis was performed, taking into account all spatial and temporal velocity measurements below the Nyquist limit and above the clutter filter cut-off, further called the normal range.

\section{CFD-based ultrasound simulations}

CFD-based US simulations were performed by coupling CFD with Field II [3], [4], which models blood as random point scatterers with normally distributed scattering amplitude. For each simulated beam, the scatterer position was updated based on the CFD-velocities, requiring spatial and temporal interpolation of the CFD-velocity vectors. Realistic carotid flow images were obtained by solving the Navier-Stokes equations (Fluent 6.2) in a patient-specific carotid artery model. We refer to [1] for further details on the CFD-US coupling procedure. In Field II, $2 \times 4 \mathrm{~cm}$ scans were simulated with a 192 element linear array transducer of $5 \mathrm{MHz}$ centre frequency (focus $=2 \mathrm{~cm}$, $\left.F_{\#, T}=2.5, F_{\#, R}=1.4\right)$. Further details on the imaging setup can be found in the ST-setup mentioned in [2].

\section{In-vivo data}

Raw IQ-data were recorded during examination of the carotid artery of a healthy volunteer using a $7 \mathrm{~L}$ vascular probe of a GE Vingmed Vivid 7 US system (GE Vingmed Ultrasound, Horten, Norway). Color flow images (CFI) were recorded with an imaging setup equal to the simulations (2 and $4 \mathrm{kHz}$ ), and the 2D flow estimates were superimposed on CFI.

\section{RESUlTS}

\section{A. CFD-based ultrasound simulations}

Fig. 1 compares the performance of ST and the three ST-PE combination techniques to the reference CFD flow field with vector plots for frame 2 (systole) and 5 (diastole). Note that the comparison is based on dynamic CFD vector fields, taking into account the ultrasonic scanning sequence. Frame 2 shows zoomed-in plots of the external carotid, the location where the highest velocities of the complete cardiac cycle prevail. A higher PRF reduces aliasing and improves ST performance. Both ST and the unwrapped version of ST-PE show a good 

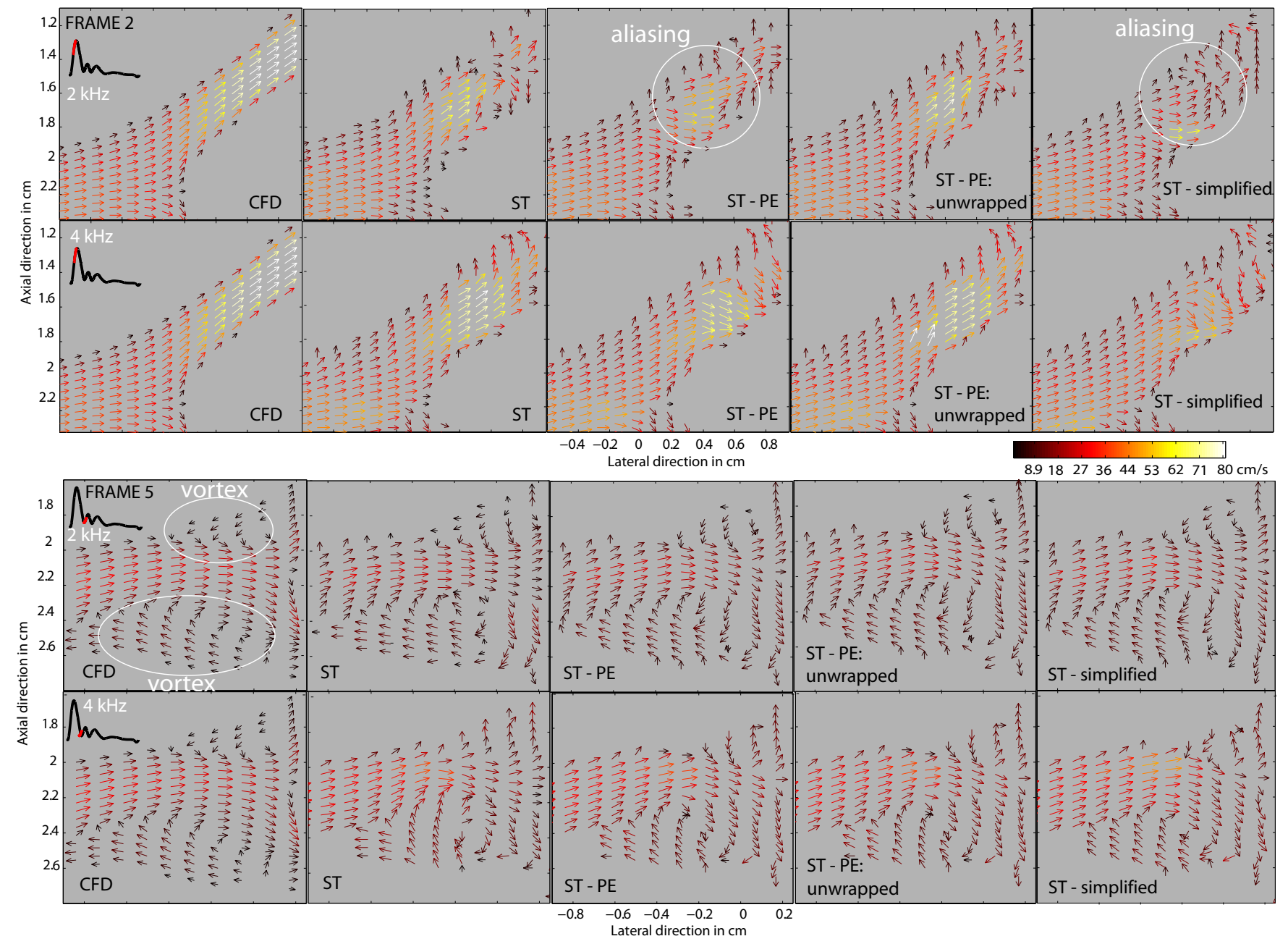

Fig. 1. Vector plots of the different 2D flow estimators compared to the CFD-reference, for systole (frame 2) and diastole (frame 5), for 2 and $4 \mathrm{kHz}$.

qualitative agreement with the reference CFD data. Integrating the PE-estimate without unwrapping (ST-PE and ST-simplified) shows obvious aliasing artifacts for both PRF's. Frame 5 shows vector plots zoomed in on the common carotid, showing a large vortex in the internal bulb and a smaller one on the opposite side. Both the 2 and $4 \mathrm{kHz}$ imaging setups are able to capture the large zone of swirling flow. Low velocities dominate during this stage and hence the influence of the clutter filter becomes important. The $4 \mathrm{kHz}$ setup estimates suffer most from clutter filtering, and all methods imaged with $4 \mathrm{kHz}$ have difficulties capturing the smaller vortex near the external carotid.

The estimator performance is further compared to the CFDreference for all available velocity estimates in space and time, using a linear regression analysis on the axial $\left(v_{z}\right)$ and lateral velocity $\left(v_{x}\right)$ component. In the normal range of velocities, a lowered spread is observed for PE estimates for all setups (Table 1 and fig.2). In fig.2, a bias is also observed for PE-variants: overestimation in the vicinity of the clutter filter transition region, while underestimation close to the Nyquist limit. Fig. 2 further shows that aliasing errors are apparent for PE but are partly corrected for by the unwrapping procedure, especially for $4 \mathrm{kHz}$. Table 1 provides the mean $(\bar{m})$ and standard deviation $(\sigma)$ on the differences between the ultrasound estimates and the reference flow, the slope $(\beta)$ and the $R^{2}$ goodness-of-fit parameter of respectively $v_{z}$ and $v_{x}$. For all imaging setups, the estimation of $v_{z}$ improves for $S T-P E$ compared to ST, in terms of decreased standard deviation $(\sigma)$

TABLE I

LINEAR REGRESSION ANALYSIS OF $v_{x}$ AND $v_{z}$ AS ESTIMATED BY ST AND THE COMBINED ESTIMATORS, COMPARED TO THE CFD REFERENCE. ANALYSIS WAS BASED ON THE NORMAL RANGE OF VELOCITIES. DATA ARE REPORTED FOR PRF $=2$ AND $4 \mathrm{KHZ}, \mathrm{SNR}=20 \mathrm{DB}$

\begin{tabular}{|cc|c|c|c|c|c|c|c|c|}
\hline & & \multicolumn{2}{|c|}{ ST } & \multicolumn{2}{c|}{ ST-PE } & \multicolumn{2}{c|}{ ST-PE:unwrap } & \multicolumn{2}{c|}{ ST-simplified } \\
\hline & & $2 \mathrm{kHz}$ & $4 \mathrm{kHz}$ & $2 \mathrm{kHz}$ & $4 \mathrm{kHz}$ & $2 \mathrm{kHz}$ & $4 \mathrm{kHz}$ & $2 \mathrm{kHz}$ & $4 \mathrm{kHz}$ \\
\hline \hline \multirow{2}{*}{$\bar{m}(\mathrm{~cm} / \mathrm{s})$} & $\mathrm{vx}$ & 2.05 & 2.69 & 2.09 & 2.75 & 2.09 & 2.72 & 1.90 & 2.28 \\
& $\mathrm{vz}$ & -0.36 & 0.36 & -0.01 & 0.21 & 0.04 & 0.63 & -0.02 & 0.10 \\
\hline \multirow{2}{*}{$\sigma(\mathrm{cm} / \mathrm{s})$} & $\mathrm{vx}$ & 5.19 & 6.32 & 5.19 & 6.27 & 5.22 & 6.38 & 5.25 & 6.70 \\
& $\mathrm{vz}$ & 2.83 & 4.67 & 2.07 & 3.48 & 2.07 & 5.46 & 2.17 & 3.55 \\
\hline \multirow{2}{*}{$\beta($ slope $)$} & $\mathrm{vx}$ & 0.86 & 0.84 & 0.86 & 0.84 & 0.86 & 0.84 & 0.86 & 0.83 \\
& $\mathrm{vz}$ & 0.92 & 0.81 & 0.95 & 0.88 & 0.94 & 0.87 & 0.96 & 0.88 \\
\hline \multirow{2}{*}{$R^{2}$ (gof) } & $\mathrm{vx}$ & 0.83 & 0.82 & 0.83 & 0.82 & 0.82 & 0.82 & 0.82 & 0.80 \\
& $\mathrm{vz}$ & 0.86 & 0.86 & 0.92 & 0.92 & 0.92 & 0.91 & 0.92 & 0.92 \\
\hline
\end{tabular}




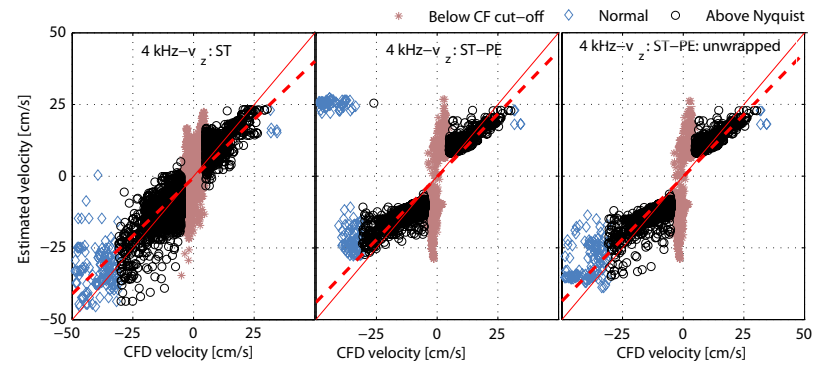

Fig. 2. Linear regression on $v_{z}$ as estimated by ST, ST-PE and STPE:unwrapped, for $4 \mathrm{kHz}$.

and an increased goodness-of-fit $\left(R^{2}\right)$. A decrease in $\sigma$ of $37 \%$ $(2 \mathrm{kHz})$ and $35 \%(4 \mathrm{kHz})$ is observed. Table 1 also quantifies a slightly deteriorated performance for $v_{x}$ of the simplified ST method.

\section{B. In-vivo data}

Fig. 3 show the ST-PE method superimposed on CFI recorded in a healthy volunteer, for frame 4 (systole) and both PRF's.

\section{DISCUSSION}

While the concept of combining ST and PE is straightforward, its practical implementation is not. Challenges arise from the fact that a common acquisition scheme does not reconcile the acquisition and processing requirements of the individual techniques. To achieve satisfactory results with the PE approach, the slow-time PRF needs to be adapted to the velocity range of interest. Further, for the combined approach to work properly, the maximum axial velocity should lie below the Nyquist limit, to avoid obscuring the combined estimate. This means that the PRF will vary for different clinical applications, and can in many cases be quite high (e.g. 4-10 kHz).

This however poses some challenges for the interleaved acquisition mode. Interleaving was used to obtain a high frame rate speckle acquisition, making tracking of complex blood movement feasible. The number of beams in each interleave group is dependent on the scan depth $\left(P R F_{\max }\right)$ and the slow-time PRF, such that high PRF's or deep scanning leads to few interleaved beams. In this case the kernel width may become very small, while the lower limit of the kernel size is determined by the imaging resolution. Hence the optimal
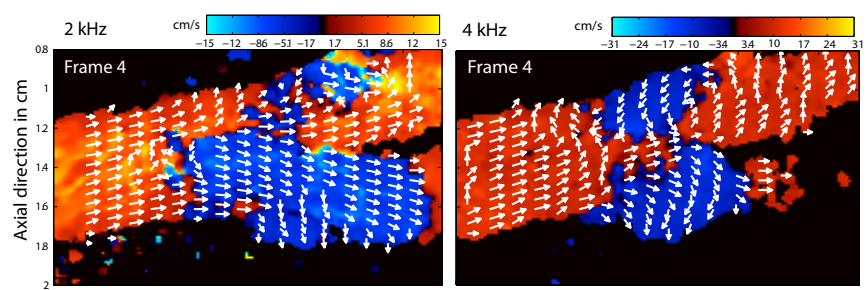

Fig. 3. ST-PE superimposed on CFI based on recordings in a healthy carotid. choice of slow-time PRF is difficult to find. In particular, adhering to a requirement of avoiding aliasing in a combined estimator might decrease performance when tracking high blood flow, compared to using ST alone at a lower PRF. Therefore, we did not only investigate a straightforward combined estimator, but also studied an unwrapping procedure based on axial ST estimates. However, due to the variance on the axial ST estimates, unwrapping may also lead to spurious errors, explaining the increased $\sigma$ of this method (Table 1). Problems also emerge when axial velocities wrap around all the way into the clutter filter stop band for low PRF's (fig.1), e.g in the external carotid during systole.

Another variant of the combined estimator was the simplified tracking approach, particularly interesting because of computational advantages compared to full 2D tracking and because potential variance attributed to $2 \mathrm{D}$ search regions can be avoided. This approach may therefore produce more stable lateral tracking estimates. However, we did not observe this due to the biased autocorrelation estimates. Interestingly, results showed that the simplified tracking approach marginally decreased lateral tracking performance (Table 1).

Overall, results showed that PE has a lower variance of $v_{z}$, compared to using pure ST for the examples explored (Table 1). One should however note a bias for both low and high velocities. Overestimation on the lower range can be attributed to the clutter filter, as polynomial regression filters are known to produce a bias close to the clutter filter transition region. The underestimation for increased velocities can partly be attributed to averaging. Finally, we illustrated the feasibility of the estimators in vivo. Fig. 3 shows that ST-PE provides reasonable velocity vector fields.

\section{CONCLUSION}

When designing a 2D velocity estimator based on ST, the axial velocity info available using the autocorrelation approach may be used to increase robustness. However, a unified acquisition which ensures good ST but also avoids aliasing is not always possible. Unwrapping using the axial ST estimate can be used, but may introduce spurious errors. A fully combined approach where only 1D lateral tracking is performed showed a marginal decrease in performance compared to $2 \mathrm{D}$ tracking, but has large computational advantages.

\section{REFERENCES}

[1] A. Swillens, L. Lovstakken, J. Kips, H. Torp, and P. Segers, "Ultrasound simulation of complex flow velocity fields based on computational fluid dynamics," IEEE Transactions on Ultrasonics, Ferroelectrics and Frequency Control, vol. 56, no. 3, pp. 546-556, 2009.

[2] A. Swillens, P. Segers, H. Torp, and L. Lovstakken, "Two-dimensional blood velocity estimation with ultrasound: speckle tracking versus crossed-beam vector doppler based on flow simulations in a carotid bifurcation model," IEEE Transactions on Ultrasonics, Ferroelectrics and Frequency Control, vol. 2, pp. 327-339, 2010.

[3] J. A. Jensen and N. B. Svendsen, "Calculation of pressure fields from arbitrarily shaped, apodized, and excited ultrasound transducers," IEEE transactions on Ultrasonics, Ferroelectrics, and Frequency control, vol. 39, no. 2, pp. 262-267, 1992.

[4] J. A. Jensen, "Field: A program for simulating ultrasound systems," Medical and Biological Engineering and Computing, vol. 34, pp. 351352, 1996. 